\title{
Simultaneous, Rapid and Nondestructive Determination of Moisture, Fat Content and Storage Time in Leisure Dried Tofu Using LF-NMR
}

\author{
Alin XIA, ${ }^{* \dagger}$ Yu Zhang, ${ }^{*}$ Liangzhong ZhaO,* and Pan QIN** \\ *College of Food and Chemical Engineering, Shaoyang University, Shaoyang 422000, China \\ **Sichuan Yijie Technology Co., Ltd, 36 Chadianzi West Street, Jinniu District, Chengdu 610036, China
}

\begin{abstract}
Leisure dried tofu is a kind of small packaged food which is popular with consumers in China. However, during the storage of leisure dried tofu, moisture and fat may be lost and deteriorate. For their own benefit, bad business operators might forge or mark the production date and shelf life. Therefore, it is necessary to explore a method to determine simultaneously the moisture, fat content, and storage time of leisure dried tofu. Samples were measured for obtaining transverse relaxation data by using low-field nuclear magnetic resonance (LF-NMR) spectrometer. The experimental data were analyzed and modeled by methods including partial least squares (PLS) or back-propagation artificial neural network (BP-ANN). The results show that the models can be used to predict the moisture, fat content, and storage time rapidly, nondestructively, accurately, and simultaneously. Furthermore, in order to explore the changes of nutrients in leisure dried tofu with the storage time, the storage dynamics of moisture and fat was considered by a using corresponding calibration model.
\end{abstract}

Keywords Leisure dried tofu, moisture, fat content, storage time, storage dynamics, LF-NMR

(Received June 23, 2020; Accepted August 26, 2020; Advance Publication Released Online by J-STAGE September 4, 2020)

\section{Introduction}

Leisure dried tofu is a kind of small package food which is made of soybean and is processed by smoking, brine, and seasoning. It is increasingly more popular among consumers because of its delicious taste and rich nutrition. ${ }^{1}$ The content and changes of the components in the leisure dried tofu are closely related to its quality. The appropriate moisture content can maintain the dried tofu concerning a good sensory properties such as flavor, appearance and morphology, and other components in the dried tofu in a balanced state, so as to reduce any deterioration due to the breeding of microorganism. As one of the most important organic nutrition macromolecules, fat is the highest calorie nutrient. Its content will affect the sensory and technological properties of leisure dried tofu. At present, drying methods and distillation methods are commonly used to obtain the moisture content value. Soxhlet extraction method is commonly used to obtain the fat content value. However, most of these traditional methods cannot meet the short-term measurement requirements of a large number of samples. They have high requirements concerning the operation of detection personnel, time-consuming and laborious, large consumption of reagents, environmental pollution and so on. In addition, during the storage process of leisure dried tofu, its moisture and fat may be lost and deteriorate with the advance of time. These content changes may also affect the quality and taste of leisure

$\dagger$ To whom correspondence should be addressed.

E-mail: alinxia@126.com dried tofu, resulting in the loss of good texture and color of the food, unpleasant smell and the loss of nutrients. ${ }^{2}$ While processing and storage, the content and change of moisture and fat are important reference indicators to measure the quality of leisure dried tofu. In order to ensure the accurate and real-time monitoring of moisture and fat changes in the storage process of leisure tofu, it is necessary to predict the content and trend of the components. Furthermore, when purchasing food, consumers usually check the production date on the product packaging to ensure that the purchased product is within the shelf life. However, for the sake of their interests, some bad businesses consciously and falsely report the production date when the goods are about to exceed the shelf life, so that consumers mistakenly think that the products they buy are fresh and qualified in quality. Such behaviors as marking false production dates and tampering with the production dates not only violate the market order and national laws and regulations, but also mislead consumers and thus their legal rights and interests are infringed. For the above reasons, there is an urgent need for a fast, nondestructive and accurate method to detect the moisture and fat in leisure dried tofu products, and to monitor the content changes during the storage process, and to predict the storage time.

Low field nuclear magnetic resonance (LF-NMR) is a new technology applied in the field of food. Compared with other technologies known at present, it has the advantages of being fast, nondestructive, and accurate, and there is no need for any chemical reagent. It has been widely used in the food industry. ${ }^{3-11}$ The detection principle is to apply RF pulses to the sample in a constant magnetic field, while producing hydrogen proton 
resonance, and then to release the absorbed RF wave energy in a non-radiative way and return to the ground state. This process is called the relaxation process, and the time constant describing the relaxation process is called the relaxation time. The relaxation time is related to the existing state of hydrogen protons as well as the physical and chemical environment. Therefore, by analyzing the relaxation time and the amplitude of the resonance signal, i.e. the LF-NMR spectrum, we can obtain much internal information concerning the sample, so as to achieve qualitative and quantitative analysis of the sample. In other words, the relaxation time and amplitude of the resonance signal are related to the moisture, fat content, and storage time of leisure dried tofu. However, the LF-NMR spectrum of leisure dried tofu has a serious overlap and a small difference, which makes it impossible to detect its components quickly using conventional methods, especially to predict the storage time. Chemometrics is an important development frontier of modern chemistry and analytical chemistry, and an important tool for a complex sample system analysis. ${ }^{12-18}$ This method has been introduced into the food field, and provides new blood for the rapid analysis of leisure dried tofu. Chemometrics can extract effective information from large and complex data more simply and quickly by means of data preprocessing or multivariate analysis. The combination of chemometrics and LF-NMR technology provides a new idea and method for the analysis of complex systems.

In this work, multivariate calibration models of moisture, fat and storage time are established using LF-NMR technology combined with partial least squares (PLS) and back-propagation artificial neural network (BP-ANN) algorithms. ${ }^{19-21}$ These models can be used to predict moisture, fat content and storage time rapidly, nondestructively, accurately and simultaneously. Furthermore, the storage dynamics of moisture and fat were studied by using a corresponding calibration model. What is more, by predicting the storage time, we can indirectly infer the production time of leisure dried tofu, which provides some reference for solving some problems, such as marking false production dates, tampering with production dates and so on.

\section{Experimental}

\section{Sample collection and reagents}

A total of 320 leisure dried tofu samples were collected from several supermarkets in Shaoyang City, Hunan Province, China in the period from September 2018 - December 2019. These samples were independent packaging of a brand with a mass of about $20-30 \mathrm{~g}$ and had a long strip shape. Petroleum ether with a boiling range of $60-90^{\circ} \mathrm{C}$ was purchased from Concord corporation (Tianjin, China). Sodium hydroxide and hydrochloric acid were purchased from Nanjing Chemical Reagent Co., Ltd (Nanjing, China). Deionized water (Millipore) was used in all experimental preparations. All reagents were of analytical grade.

\section{LF-NMR measurements}

The leisure dried tofu samples to be tested did not need any pretreatment and were directly packed in cylindrical glass tubes (26 $\mathrm{mm}$ in diameter). It was placed in the measuring cell of a $23 \mathrm{MHz}$ LF-NMR analyzer (MQC-23, Oxford Instruments plc, UK) and preheated for $10 \mathrm{~min}$ before a measurement. The temperature of the instrument magnet was $32^{\circ} \mathrm{C}$. Signal acquisition was carried out using the CPMG (Carr-PurcellMeiboom-Gill) pulse sequence. The main parameters were set as follows: $P_{90^{\circ}}\left(90^{\circ}\right.$ pulse time), $5.45 \mu \mathrm{s} ; P_{180^{\circ}}\left(180^{\circ}\right.$ pulse time),
$10.9 \mu \mathrm{s} ; \mathrm{SW}$ (sweep width), $1000 \mathrm{kHz}$; RD (repetition delay), $1000 \mathrm{~ms}$; NS (number of scans), 16; NECH (number of echoes), 512; TAU (pulse gap), $150 \mu \mathrm{s}$. The measured echo curve was inverted to the transverse relaxation time spectrum $\left(T_{2}\right.$ spectrum). All measurements were performed with three repetitions for each sample. The mean value was taken as the measurement result. The $T_{2}$ spectrum was a curve obtained from the inversion of the multiple exponential relaxation spectrum of the echo signal by using inversion algorithm run by a WinDxp software of LF-NMR analyzer. The default output of the spectral data was 128 data points.

\section{Reference value}

GB5009.3-2016 (Chinese national standard) was used to measure the moisture content. The samples were dried at $105^{\circ} \mathrm{C}$ in an electric blast drying oven (101-1AB, Tianjin taist instrument, China) until the weight difference was reduced to less than $2 \mathrm{mg}$.

The acid hydrolysis method in GB 5009.6-2016 (Chinese national standard) was used to measure the fat content. After acid hydrolysis of the samples with hydrochloric acid, petroleum ether was used for extraction. The fat (free and combined) content in leisure dried bean was obtained by removing the solvent. An electronic balance (CP213, Aohaus Corporation, USA) was used to weigh the fat.

The starting point of the storage time was the production date identified on the package. The storage time was in days. All samples were stored at room temperature. The samples needed to be measured by a LF-NMR to obtain spectra before a reference value analysis.

\section{Statistical analysis}

Partial least squares (PLS) and back-propagation artificial neural networks (BP-ANN) programs were in-house written and run in the Octave software platform. The optimal principal component number of the PLS model was obtained by using the leave-one-out cross-validation method. ${ }^{22,23}$

In order to measure the predictive performance of an obtained model, the root-mean-squared errors of the calibration (RMSEC) and the root-mean-squared errors of the prediction (RMSEP) were introduced and defined respectively as ${ }^{24,25}$

$$
\begin{aligned}
& \text { RMSEC }=\sqrt{\frac{\sum_{\mathrm{i}=1}^{\mathrm{n}}\left(\hat{y}_{\mathrm{i}}-y_{\mathrm{i}}\right)^{2}}{n},} \\
& \mathrm{RMSEP}=\sqrt{\frac{\sum_{\mathrm{i}=1}^{\mathrm{n}}\left(\hat{y}_{\mathrm{i}}-y_{\mathrm{i}}\right)^{2}}{m} .}
\end{aligned}
$$

where $n$ and $m$ denoted the number of calibration and prediction sets samples respectively, $\hat{y}_{\mathrm{i}}$ is the predicted value for the $i$ th sample, and $y_{\mathrm{i}}$ is the known concentration for the $i$ th sample.

All calculations were carried out on a personal computer with an Intel Core i5-8400 processor and 8GB RAM under the Windows 10 operating system. Plotting diagrams were generated using an Origin 75 (OriginLab Corporation, Northampton, MA, USA).

\section{Results and Discussion}

\section{Quantitative models}

Reference value analysis. After 104 and 92 samples were randomly selected from all 320 samples for establishing 
Table 1 Statistics of measured reference values of moisture and fat

\begin{tabular}{llcccc}
\hline Component & \multicolumn{1}{c}{ Data set } & Number & Min & Max & Mean $\pm \mathrm{SD}^{\mathrm{a}}$ \\
\hline Moisture & Calibration set & 77 & 0.2900 & 0.8768 & $0.5916 \pm 0.1239$ \\
& Test set & 27 & 0.2988 & 0.8800 & $0.6019 \pm 0.1352$ \\
Fat (g/100 g) Calibration set & 69 & 5.5147 & 9.9601 & $8.3295 \pm 1.2037$ \\
& Test set & 23 & 5.5150 & 9.9580 & $8.3236 \pm 1.2408$
\end{tabular}

a. $\mathrm{SD}$ is the standard deviation

calibration models of moisture and fat contents, respectively. The samples were used for reference value analysis after LFNMR measurements. Statistics of measured reference values of moisture and fat content were shown in Table 1. According to the analysis results in Table 1 , the reference values of moisture and fat content were distributed in the range of $0.29-0.88$, and in the range of $5.5147-9.9601 \mathrm{~g} / 100 \mathrm{~g}$, respectively. The ranges of moisture and fat contents basically covered the possible contents in the leisure dried tofu, so the samples had representativeness for establishing calibration models.

Moisture calibration model. First 77 samples were randomly selected from 104 samples for a calibration set. The remaining 27 samples were used for a test set. The optimal principalcomponent number of the PLS model was 6 by using the leaveone-out cross-validation method. The PLS method was used to establish the calibration model for 77 calibration samples of dried soybeans. The accuracy of the model was tested by 27 validation samples that did not participate in the modeling. Figure 1a shows the relationship between the predicted value of the model and the actual measured value. As shown in Fig. 1a, there was a good correlation between the predicted moisture content value and the actual measured value through the PLS model. BP-ANN was also used to establish a multivariate calibration model for calibration set of dried tofu samples. After learning and training by the Levenberg-Marquardt optimization method, it was determined that BP-ANN with a single hidden layer was used as a multivariate calibration model. The model contained an input layer with 128 nodes (i.e. $\mathrm{T}_{2}$ points), an output layer with 1 node and an implicit layer. The number of implicit layer nodes can be determined as follows by reference to an empirical formula: $n=\sqrt{a \times b}$, where $a$ and $b$ denote the number of input and output nodes, respectively. Thus the number of implicit layer nodes was 11 . The accuracy of the BP-ANN calibration model was tested by 27 validation samples which were not involved in the modeling. The results are given in Fig. 1b. It can be seen from Figure $1 b$ that there was also a good correlation between the predicted moisture content value and the actual measured value through the BP-ANN model.

Table 2 shows the results of the two methods. It can be seen from Table 2 that RMSEC and RMSEP were both low $(<0.05)$, and the correlation coefficient of the calibration set $\left(R_{\mathrm{C}}\right)$ and of the test set $\left(R_{\mathrm{P}}\right)$ were both high $(>0.9)$ by PLS and BP-ANN models. In general, the performance of the BP-ANN model is slightly better than that of the PLS model. This might be because the linear relationship between the moisture content and transverse relaxation spectra was not very strong. Nevertheless, the BP-ANN method was generally superior to the PLS method in the quantitative analysis of nonlinear data. In other words, the better is the linearity of the analysis data, the better is the prediction effect of the PLS method; the worse is the linearity, the better is the prediction effect of the BP-ANN method. All in all, the results showed that the quantitative prediction models based on LF-NMR combined with the two methods had high
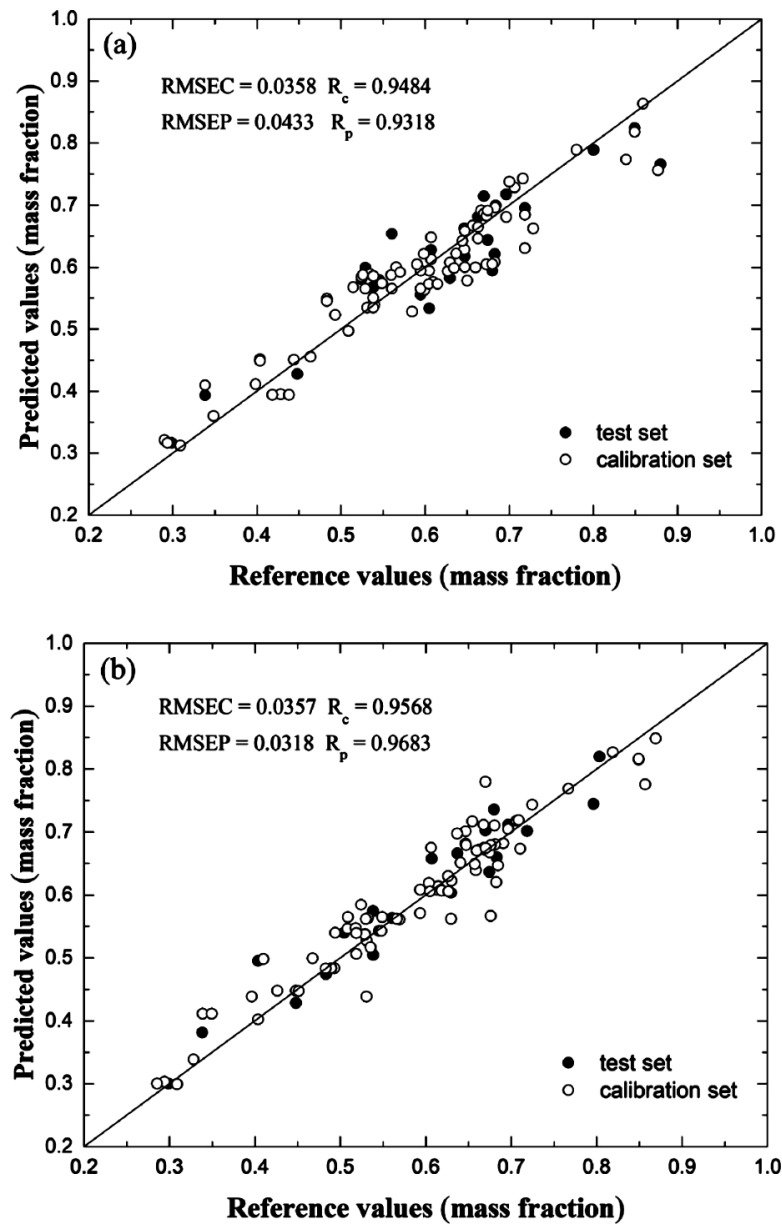

Fig. 1 Correlation between reference and predicted values of the moisture content for PLS (a) and BP-ANN (b).

Table 2 Statistical results of predicting the moisture content of leisure dried tofu by PLS and BP-ANN

\begin{tabular}{lcccc}
\hline Method & RMSEC & $R_{\mathrm{C}}$ & RMSEP & $R_{\mathrm{P}}$ \\
\hline PLS & 0.0358 & 0.9484 & 0.0433 & 0.9318 \\
BP-ANN & 0.0357 & 0.9568 & 0.0318 & 0.9683 \\
\hline
\end{tabular}

accuracy and good prediction ability. It can be used to replace the traditional chemical method for a nondestructive determination of the water content in leisure dried tofu, and can ensure the accuracy requirements of the conventional analysis method. Since the BP-ANN model was better than the PLS model, the BP-ANN model was chosen and used for subsequent dynamic analysis of the moisture content in dried tofu.

Fat calibration model. After 69 samples were randomly selected from 92 samples for the calibration set, the remaining 23 samples were used for the test set. The optimal principalcomponent number of the PLS model was 9 by using the leaveone-out cross-validation method. The PLS method was used to establish the calibration model for 69 calibration samples of leisure dried tofu. The accuracy of the model was tested by 23 validation samples that did not participate in the modeling. Figure 2a shows the relationship between the predicted value of the model and the actual measured value. As shown in Fig. 2a, there was a good correlation between the predicted fat content 

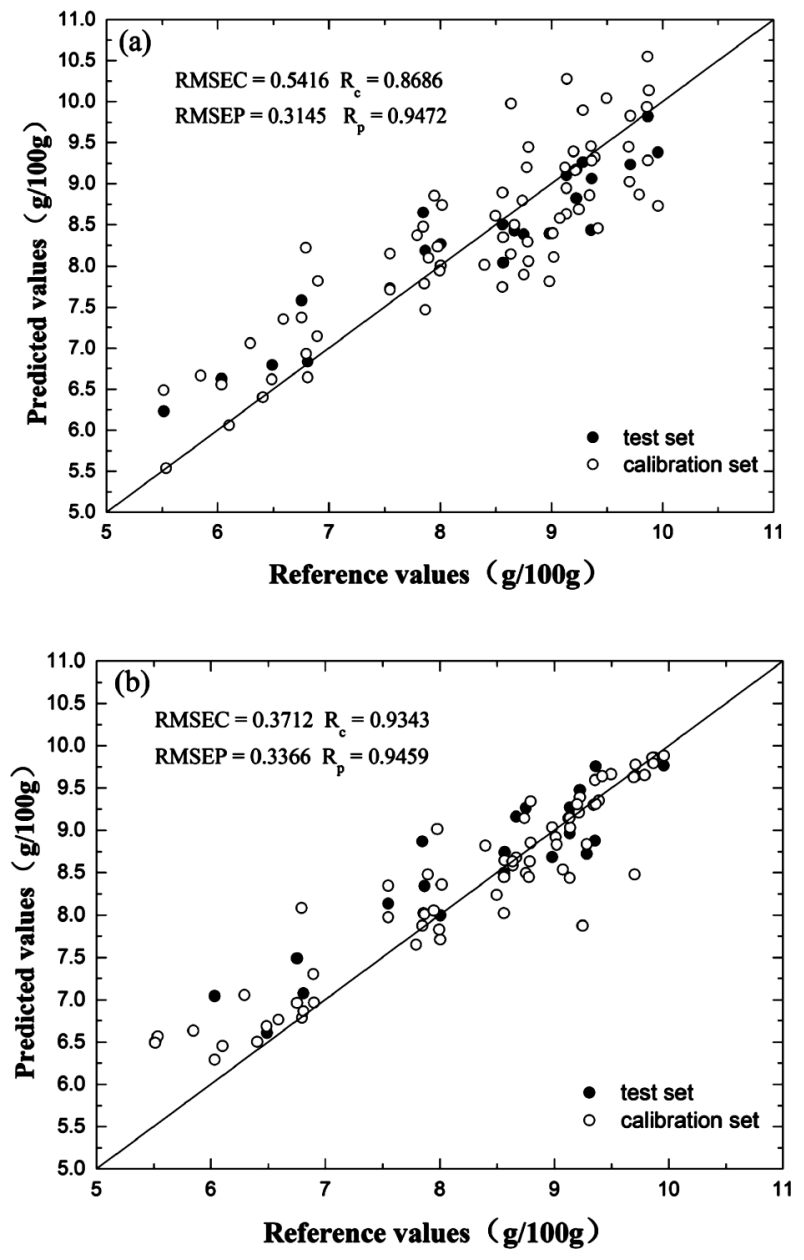

Fig. 2 Correlation between the reference and predicted values of the fat content for PLS (a) and BP-ANN (b).

Table 3 Statistical results of predicting the fat content of leisure dried tofu by PLS and BP-ANN

\begin{tabular}{lcccc}
\hline \multicolumn{1}{c}{ Method } & RMSEC & $R_{\mathrm{C}}$ & RMSEP & $R_{\mathrm{P}}$ \\
\hline PLS & 0.5416 & 0.8686 & 0.3145 & 0.9472 \\
BP-ANN & 0.3712 & 0.9343 & 0.3366 & 0.9459 \\
\hline
\end{tabular}

value and the actual measured value through the PLS model. BP-ANN was also used to establish the fat calibration model for the calibration set of dried tofu samples by reference to the method in the section "Moisture calibration model". The accuracy of the BP-ANN calibration model was tested using 23 validation samples which were not involved in the modeling. The results were shown in Fig. $2 \mathrm{~b}$. It can be seen from the figure that there was also a good correlation between the predicted fat content value and the actual measured value through BP-ANN model.

Table 3 shows the results of the two methods. It can be seen from Table 3 that the PLS and BP-ANN models, respectively, gave RMSEC values of 0.5416 and 0.3712 , and $R_{\mathrm{C}}$ values of 0.8686 and 0.9343 . RMSEC obtained by BP-ANN was obviously smaller than that by PLS, and $R_{\mathrm{C}}$ obtained by BPANN was higher than that by PLS. This showed that the performance of the BP-ANN model was obviously better than that of the PLS model. This might be due to the weak linear relationship between fat content and the transverse relaxation spectra. In addition, RMSEP and $R_{\mathrm{P}}$ obtained by the PLS and BP-ANN models were close to each other. This showed that although the performance of PLS model was not very good, it could also get good prediction results. For predicting the test set, both methods could obtain high precision and good prediction ability. In general, the BP-ANN method was more robust in predicting fat content of leisure dried tofu compared with the PLS method. Therefore, the following dynamic analysis of the fat content was carried out by using the BP-ANN model.

\section{Dynamics analysis}

Dried tofu moisture dynamics. After 20 samples were randomly selected from all 320 samples, within about 400 days, a LFNMR analyzer was used to measure the samples every other period of time. The BP-ANN model calibration established in the section "Moisture calibration model" was used to predict the moisture content of these samples. Figure 3 is the scatter diagram of the relationship between the storage time and the predicted moisture content of 20 leisure dried tofu samples. Figures $3 \mathrm{a}, 3 \mathrm{~b}, 3 \mathrm{c}$, and $3 \mathrm{~d}$ correspond to samples $1-5,6-10$, $11-15$ and samples $16-20$, respectively. In Fig. 3 , the $x$-axis is the storage time, the $y$-axis is the sample number, and the $z$-axis is the predicted value of the moisture content obtained from the BP-ANN model established by the above study. As shown in Fig. 3, in about 400 days, the moisture content value of leisure dried tofu showed a fluctuating downward trend with an extension of the storage time. It is worth mentioning that the predicted moisture content values are based on the reference values obtained by the atmospheric drying method. Therefore, the decrease in the moisture content did not mean that water would escape from the sample and disappear. This might be due to the fact that the water in dried tofu gradually reacted with other substances or transformed into tightly bound water with the extension of the storage time, resulting in the gradual decrease of the moisture content.

Dried tofu fat dynamics. After 20 samples were randomly selected from all 320 samples, within about 400 days, a LFNMR analyzer was used to measure the samples every other period of time. These samples were predicted by the BP-ANN model established in the section called "Fat calibration model". Figure 4 is a scatter diagram of the relationship between the storage time and the predicted fat content of 20 leisure dried tofu samples. Figures $4 \mathrm{a}, 4 \mathrm{~b}, 4 \mathrm{c}$ and $4 \mathrm{~d}$ correspond to samples $1-5,6-10,11-15$ and samples $16-20$, respectively. As shown in Fig. 4, the fat content of all leisure dried tofu samples remained stable for about 180 days (about 6 months). However, with the prolongation of the storage time, the fat content of most samples showed obvious fluctuation after 180 days. This might have been due to the chemical reaction of fat and other substances in dried tofu with the extension of the storage time, leading to the fluctuation of fat content. This kind of fat reaction may be the beginning of tofu spoilage. This change might reflect the shelf life of dried tofu. Therefore, it could be suggested to the manufacturer that the shelf life of leisure dried tofu should be marked as 6 months.

\section{Storage time calibration model}

First 40 samples were randomly chosen from all 320 samples. Under the same conditions, the samples were measured with a LF-NMR instrument every few days within about 400 days. The spectra were collected and the days of the storage time were recorded. In this way, a total of 1040 spectra were obtained. Each spectrum was regarded as a sample, i.e. 1040 

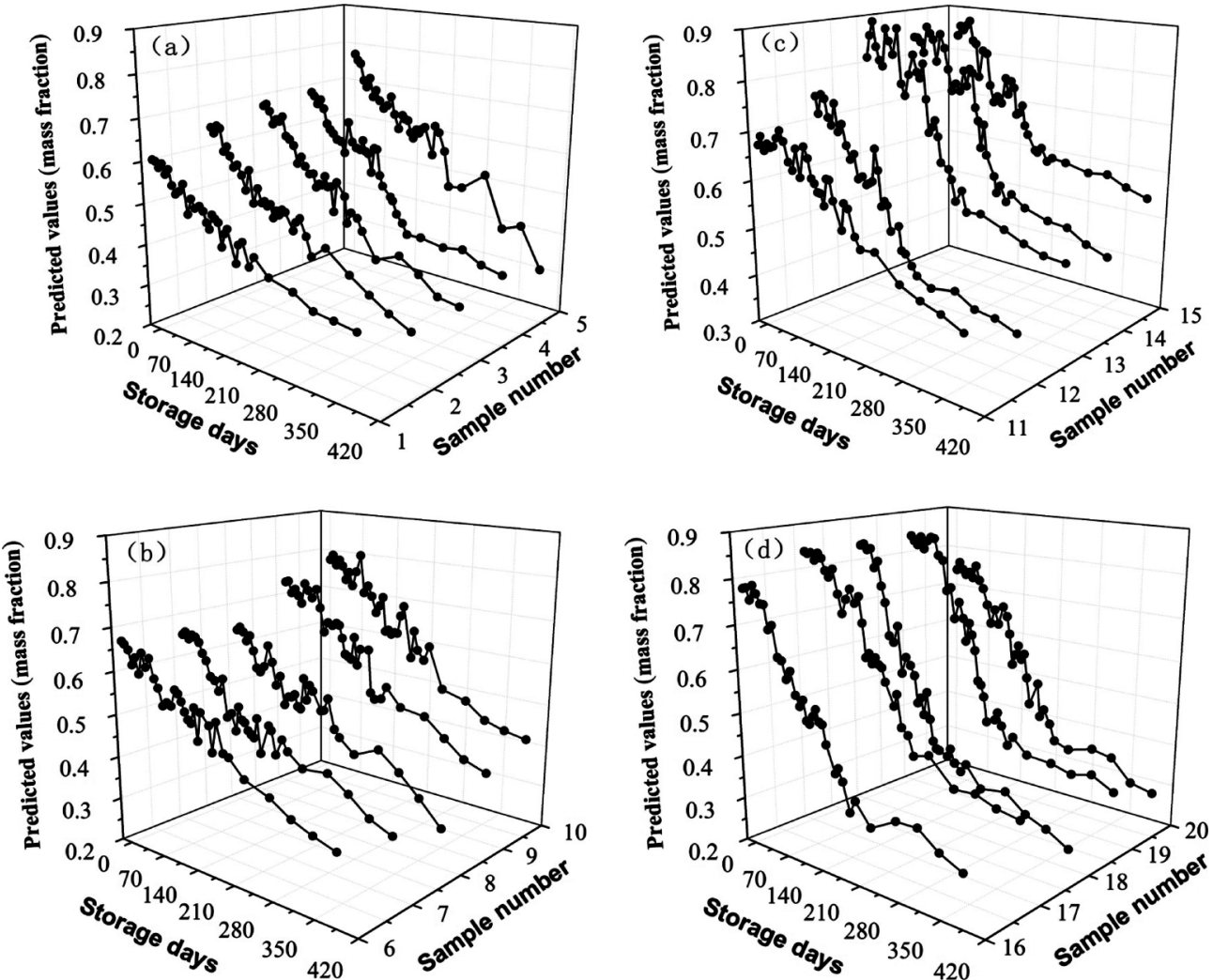

Fig. 3 Relationship between the storage time and predicted moisture content for samples $1-5$ (a), samples 6 - 10 (b), samples $11-15$ (c) and samples $16-20$ (d). The $x$-axis is number of storage days, the $y$-axis is the sample number, and the $z$-axis is the predicted value of the moisture content.
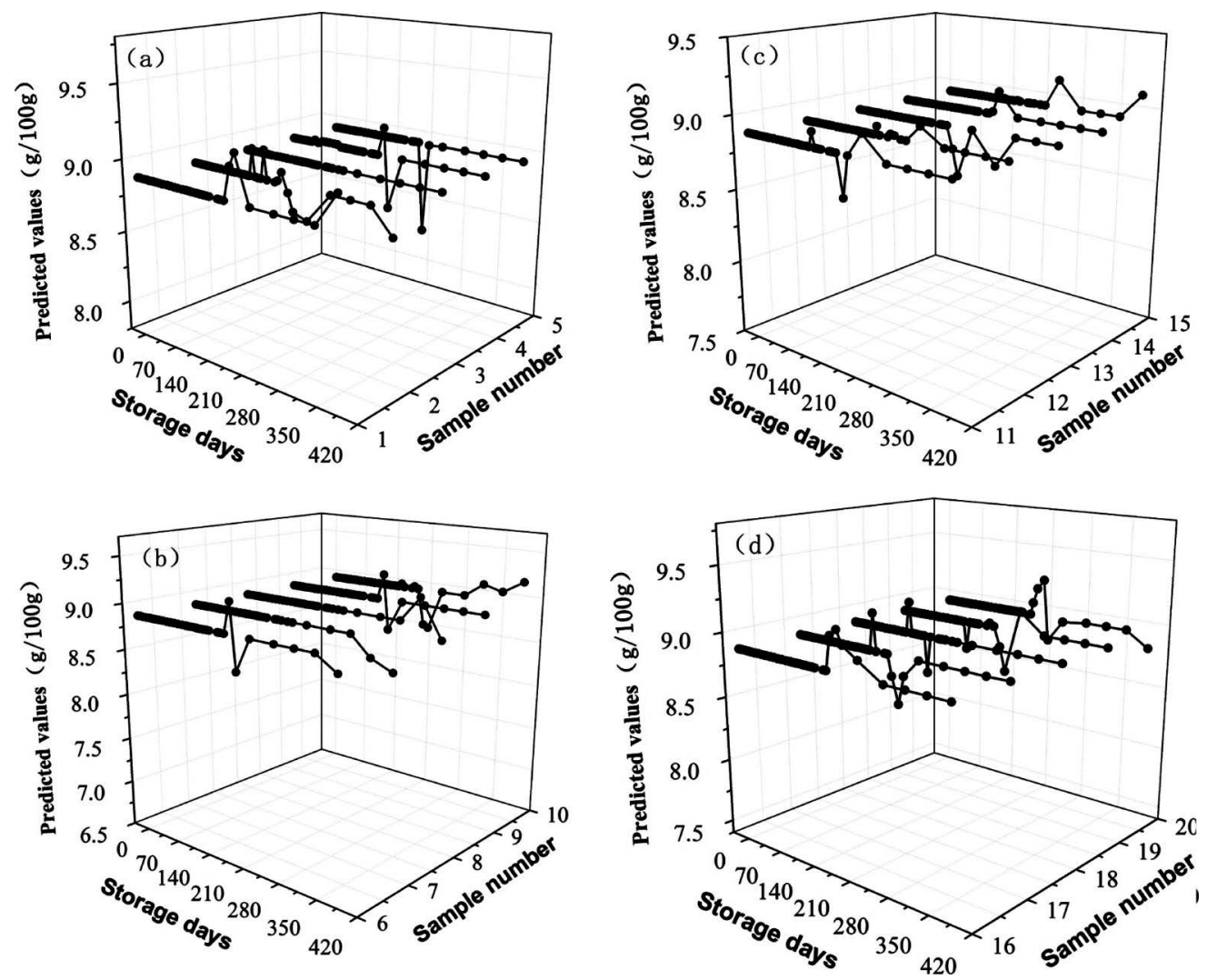

Fig. 4 Relationship between the storage time and the predicted fat content for samples $1-5$ (a), samples 6 - 10 (b), samples 11 - 15 (c) and samples 16 - 20 (d). 
samples; 780 samples were randomly selected from 1040 samples for the calibration set. The remaining 260 samples were used for the test set. BP-ANN was also used to establish a multivariate calibration model for a calibration set of dried tofu samples. The reason why the PLS method was not selected was that the result of PLS modeling was very bad. This might be due to the strong nonlinear relationship between the storage time and the transverse relaxation spectrum. In general, the BPANN method was better than the PLS method for analyzing nonlinear data. The Bayesian regularization method was used as the training function of the model..$^{26,27}$ The learning function adopted the learning function of the weight value and the offset value of the gradient of the driving quantity. The tangent transfer function of hyperbola was chosen as the transfer function of a hidden layer, and the linear function was chosen as the transfer function of the output layer. The model contained an input layer with 128 nodes (i.e. $\mathrm{T}_{2}$ points), and an output layer with 1 node. The number of an implicit layer nodes can be determined as 11 by reference to an empirical formula in the section "Moisture calibration model". The accuracy of the BPANN calibration model was tested by 260 validation samples which were not involved in the modeling. The results are shown in Fig. 5, which shows that there was a good correlation between the actual and predicted days through the BP-ANN model. RMSEC and RMSEP were 26.65 and 29.92, respectively, which

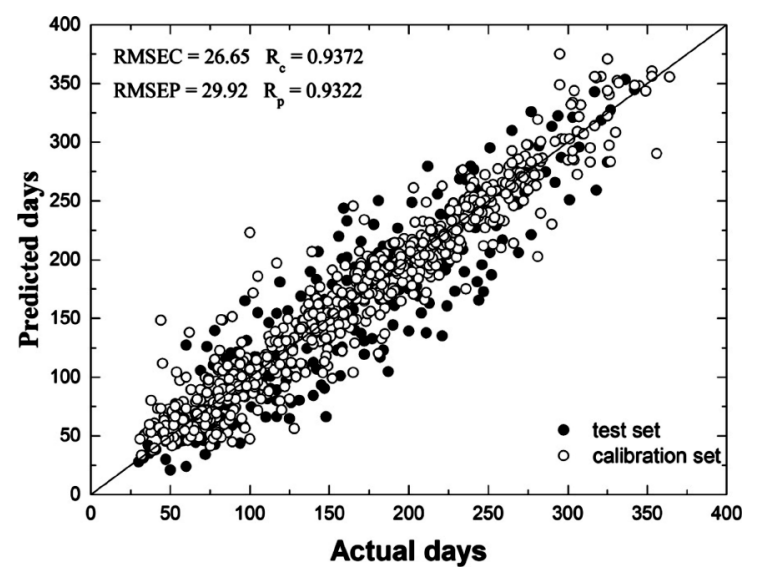

Fig. 5 Correlation between the actual and predicted days for BP-ANN. were less than 30 , indicating that the average error of prediction days was less than 1 month. $R_{\mathrm{C}}$ and $R_{\mathrm{P}}$ were 0.9375 and 0.9322 , respectively, both higher than 0.9 , with a high degree of correlation. So the model had a high prediction accuracy and a good prediction ability, which can be used to predict the storage time of leisure dried tofu.

Simultaneous prediction of moisture, fat content and storage time Twelve samples were randomly selected from all 320 samples for the prediction set. BP-ANN calibration models were chosen from the calibration models established in the sections "Moisture calibration model", "Fat calibration model" and "Storage time calibration model" to predict the moisture content, fat content and storage time, respectively. Table 4 shows the predicted results of prediction set samples using the BP-ANN calibration models. As shown in Table 4, the relative errors of the predicted results of the moisture and fat content models were almost less than $10 \%$ compared with the reference values. The relative errors of the storage time model were mostly less than $10 \%$, and the maximum error was less than $30 \%$. There seemed to be a big error in this result. There were three main reasons for this big error. One was the fluctuation of the sample temperature, which affected the transverse relaxation, leading to spectral fluctuation, and resulting in poor repeatability. In a 400-day time span, the sample temperature still fluctuated with the weather, although in an air-conditioned environment. The second was the uniformity of the sample. In the process of stewing and packaging dried tofu, it was difficult to ensure that the components of each small package of dried tofu were uniform. The samples used for testing were measured together with the packaging, so it would affect the analysis results. The last point was that the correlation between the storage time and the resonance signal was weak, which made the prediction of the storage time greatly affected by the fluctuation of the resonance signal. However, the storage time has always been a difficult parameter to predict. The results were thus also acceptable. On the whole, the prediction performance of the calibration models was excellent, and the predicted results were basically close to the reference or actual values.

\section{Conclusions}

BP-ANN and PLS methods combined with LF-NMR were used to establish corresponding prediction models for the moisture

Table 4 Predicted results of prediction set samples using BP-ANN models

\begin{tabular}{|c|c|c|c|c|c|c|c|c|c|}
\hline \multirow{2}{*}{ Sample } & \multicolumn{3}{|c|}{ Moisture content } & \multicolumn{3}{|c|}{ Fat content $(\mathrm{g} / 100 \mathrm{~g})$} & \multicolumn{3}{|c|}{ Day } \\
\hline & $\operatorname{Ref}^{a}$ & Pre $^{b}$ & $\mathrm{RE}^{\mathrm{c}}, \%$ & Ref & Pre & RE, \% & Actual & Pre & $\mathrm{RE}, \%$ \\
\hline 1 & 0.5916 & 0.6101 & 3.13 & 6.4912 & 6.6101 & 1.83 & 128 & 121 & -5.47 \\
\hline 2 & 0.5481 & 0.5142 & -6.19 & 9.2243 & 9.4785 & 2.76 & 161 & 175 & 8.70 \\
\hline 3 & 0.6463 & 0.6814 & 5.43 & 6.7537 & 7.4873 & 10.86 & 86 & 97 & 12.79 \\
\hline 4 & 0.6704 & 0.7025 & 4.79 & 9.1370 & 9.2691 & 1.45 & 110 & 98 & -10.91 \\
\hline 5 & 0.7182 & 0.7011 & -2.38 & 9.2845 & 8.7229 & -6.05 & 52 & 67 & 28.85 \\
\hline 6 & 0.4833 & 0.4746 & -1.80 & 7.5508 & 8.1335 & 7.72 & 135 & 126 & -6.67 \\
\hline 7 & 0.7375 & 0.7663 & 3.91 & 9.8674 & 9.8593 & -0.08 & 75 & 82 & 9.33 \\
\hline 8 & 0.5075 & 0.5477 & 7.92 & 8.7522 & 9.2651 & 5.86 & 168 & 182 & 8.33 \\
\hline 9 & 0.4291 & 0.4037 & -5.92 & 8.0049 & 7.9946 & -0.13 & 326 & 307 & -5.83 \\
\hline 10 & 0.5629 & 0.5728 & 1.76 & 7.8688 & 8.3427 & 6.02 & 195 & 215 & 10.26 \\
\hline 11 & 0.6067 & 0.6075 & 0.13 & 5.9159 & 6.5032 & 9.93 & 217 & 202 & -6.91 \\
\hline 12 & 0.5088 & 0.5123 & 0.69 & 6.4913 & 6.6094 & 1.82 & 180 & 189 & 5.00 \\
\hline
\end{tabular}

a. Ref is the reference value. b. Pre is the predicted value. c. RE is the relative error. 
content, fat content and storage time of leisure dried tofu. The results showed that the BP-ANN prediction model of the moisture content was slightly better than the PLS model. The BP-ANN model was obviously better than the PLS model in predicting the fat content. Because of the poor performance of the storage time prediction model established by the PLS method, the BP-ANN method was used for modeling. The BPANN model gave RMSEC, RMSEP, $R_{\mathrm{C}}$ and $R_{\mathrm{P}}$ values of 26.65 , $29.92,0.9375$ and 0.932 , respectively. As the prediction of the storage time is always a difficult problem, the performance of the prediction model is satisfactory. Furthermore, the BP-ANN model of the moisture content, fat content and storage time was used to predict the moisture content, fat content and storage time of 12 leisure dried tofu samples. The prediction value was basically close to the reference value or the actual value. In addition, the corresponding calibration models were used to analyze the dynamics of water and fat. The moisture content of leisure dried tofu fluctuated and decreased with an extension of the storage time by about 400 days. The fat content of most samples showed obvious fluctuations after 180 days. This change might reflect the shelf life of dried tofu. It is suggested that manufacturers should mark the shelf life of leisure dried tofu as being 6 months. All in all, this study can provide some ideas and methods for comprehensive and accurate evaluations of the quality and safety of leisure dried tofu. It can provide a reference for solving some problems, such as marking a false production date, tampering with the production date and so on, as well as the problems that it is unable to monitor the quality of leisure dried tofu in a fast, effective, nondestructive and realtime way in the storage process. It also provides some theoretical and technical support for improving the quality assurance and supervision system of leisure dried tofu.

\section{Acknowledgements}

The authors acknowledge financial support of Key Scientific Research Projects of Hunan Provincial Department of Education of China (Grant No. 16A236) and Research and Innovation Project for Postgraduates of Shaoyang University (Grant No. CX2019SY052).

\section{References}

1. M. Kim, I. S. Son, and J. S. Han, J. Food Quality, 2004, 27, 27.

2. M. Zhao, C. Esquerre, G. Downey, and C. P. O'Donnell, Food Control, 2017, 73, 1082.
3. T. Li, X. Rui, W. Li, X. H. Chen, M. Jiang, and M. S. Dong, J. Agric. Food Chem., 2014, 62, 8594.

4. I. Sanchezalonso, P. Moreno, and M. Careche, Food Chem., 2014, 153, 250.

5. L. Chen, Y. Q. Tian, B. H. Sun, J. P. Wang, Q. Y. Tong, and Z. Y. Jin, Food Chem., 2017, 233, 525.

6. Q. Sun, M. Zhang, and P. Q. Yang, LWT-Food Sci. Technol., 2019, 116, 108548.

7. J. P. Renou, J. Kopp, P. Gatellier, G. Monin, and G. KozakReiss, Meat Sci., 1989, 26, 101.

8. H. C. Bertram, Magn. Reson. Imaging, 2004, 22, 557.

9. Y. Sangpring, M. Fukuoka, N. Ban, H. Oishi, and N. Sakai, J. Food Eng., 2017, 211, 7.

10. C. S. Carneiro, E. T. Mársico, R. O. Ribeiro, C. A. ConteJúnior, S. B. Mano, C. J. C. Augusto, and E. F. O. Jesus, J. Food Eng., 2016, 169, 321.

11. M. Defernez, E. Wren, A. D. Watson, Y. Gunning, I. J. Colquhoun, G. L. Gall, D. Williamson, and E. K. Kemsley, Food Chem., 2017, 216, 106.

12. K. S. Booksh and B. R. Kowalski, Anal. Chem., 1992, 66, 782.

13. M. Ahmadvand, H. Parastar, H. Sereshti, A. Olivieri, and R. Tauler, Anal. Chim. Acta, 2017, 952, 18.

14. D. C. Maira, A. A. Juan, and M. E. Graciela, Talanta, 2017, 165,52

15. A. L. Xia, H. L. Wu, D. M. Fang, Y. J. Ding, L. Q. Hu, and R. Q. Yu, Anal. Sci., 2006, 22, 1189.

16. A. L. Xia, H. L. Wu, S. H. Zhu, Q. J. Han, Y. Zhang, and R. Q. Yu, Anal. Sci., 2008, 24, 1171.

17. S. Morita, Anal. Sci., 2020, 36, 107.

18. Y. Morisawa, Anal. Sci., 2019, 35, 833.

19. Z. Genisheva, C. Quintelas, D. P. Mesquita, E. C. Ferreira, J. M. Oliveira, and A. L. Amaral, Food Chem., 2018, 246, 172.

20. P. S. Sampaio, A. Soares, A. Castanho, A. S. Almeida, J. Oliveira, and C. Brites, Food Chem., 2018, 242, 196.

21. M. Khayet and C. Cojocaru, Sep. Purif. Technol., 2012, 86, 171.

22. G. C. Cawley and N. L. C. Talbot, Neural Networks, 2004, 17, 1467.

23. S. Wold, M. Sjostrom, and L. Eriksson, Chemom. Intell. Lab. Syst., 2001, 58, 109.

24. A. Lorber, K. Faber, and B. R. Kowalski, Anal. Chem., 1997, 69, 1620.

25. H. A. Martens and P. Dardenne, Chemom. Intell. Lab. Syst., 1998, 44, 99

26. D. J. C. Mackay, Neural Comput., 1992, 4, 415.

27. K. Duan, W. H. Xiao, Y. D. Mei, and D. D. Liu, Stoch. Env. Res. Risk A, 2014, 28, 1985. 